described in this brief discussion, it is found that the third integer is the sum of three biquadrates, and gives Norrie's solution

$$
d=198593=\frac{353^{4}-272^{4}}{15^{4}}=21^{4}+8^{4}+2^{4} .
$$

The seventh integer furnishes a second numerical solution

$$
d=5086913=\frac{651^{4}-599^{4}}{10^{4}}=43^{4}+34^{4}+24^{4} .
$$

It is the intention of the writer to carry the investigation of each case as far as values of $x<1250\left(=2 \cdot 5^{4}\right)$. The fact that an additional solution was obtained in the first case examined would seem to give some promise that other solutions exist among the lower integers.

Glenside, Pa.

\title{
ON THE INVERSION OF THE $q$-SERIES ASSOCIATED WITH JACOBIAN ELLIPTIC FUNCTIONS
}

A. N. LOWAN, G. BLANCH AND W. HORENSTEIN ${ }^{1}$

The elliptic functions sn $(u, k)$, cn $(u, k)$ and $\mathrm{dn}(u, k)$ may be computed from theta functions by well known methods outlined in standard texts. (See, for instance, Whittaker \& Watson, Modern Analysis, 4 th edition, p. 485.) Given the modulus $k$, and $k^{\prime}=\left(1-k^{2}\right)^{1 / 2}$, there is associated with $k, k^{\prime}$ a function $\epsilon$ defined by

$$
\epsilon=\frac{1}{2}\left[\frac{1-\left(k^{\prime}\right)^{1 / 2}}{1+\left(k^{\prime}\right)^{1 / 2}}\right]=\frac{1}{2} \frac{\vartheta_{2}\left(0, q^{4}\right)}{\vartheta_{3}\left(0, q^{4}\right)} .
$$

Values of theta functions for a given parameter $q$ can be readily computed, and the Jacobi elliptic functions turn out to be ratios of the theta functions.

The series for $\epsilon$ in terms of $q$ is given by

$$
\epsilon=\sum_{k=0}^{\infty} q^{(2 k+1)^{2}} / 1+2 \sum_{k=1}^{\infty} q^{4 k^{2}} .
$$

Presented to the Society, February 28, 1942; received by the editors December 19, 1941.

${ }^{1}$ The above results were obtained in the course of work carried out by the Mathematical Tables Project, conducted by the Work Projects Administration for the City of New York under the sponsorship of the National Bureau of Standards. 
Since, however, the known parameter in elliptic functions is $\epsilon$ and not $q$, the problem of inverting formula (1) arises. Weierstrass ${ }^{2}$ gave the first four terms of this expansion, and Milne-Thomson ${ }^{3}$ added two more, giving

$$
q=\epsilon+2 \epsilon^{5}+15 \epsilon^{9}+150 \epsilon^{13}+1707 \epsilon^{17}+20,910 \epsilon^{21}+\cdots .
$$

As is well known, the above series converges for $|\epsilon|<1 / 2$; and it is rapidly convergent over a large range of $\epsilon$. But when $\epsilon$ is greater than 0.3 , only a few decimals in $q$ may be obtained from the six terms given above. Because of the importance of this series in the computation of elliptic functions as well as in the Jacobian zeta functions, the above series has been extended to fourteen terms, as given below:

$$
\begin{aligned}
q= & \epsilon\left[1+2 u+15 u^{2}+150 u^{3}+1707 u^{4}+20,910 u^{5}+268,616 u^{6}\right. \\
& +3,567,400 u^{7}+48,555,069 u^{8}+673,458,874 u^{9} \\
& +9,481,557,398 u^{10}+135,119,529,972 u^{11} \\
& \left.+1,944,997,539,623 u^{12}+28,235,172,753,886 u^{13} \cdots\right]
\end{aligned}
$$

where $u=\epsilon^{4}$.

The following accuracy may be obtained with the above series:

$\begin{array}{ccc} & \text { Accuracy in } q \text { with } & \text { Accuracy in } q \text { with } \\ \epsilon & 14 \text { terms of }(2) & \text { terms of }(2) \\ & \text { decimals } & \text { decimals } \\ .4 & 7 & 4 \\ .36 & 10 & 5 \\ .30 & 14 & 6 \\ .25 & 18 & 8\end{array}$

New York City

${ }^{2}$ Werke, vol. 2 (1895), p. 276.

${ }^{3}$ Ten decimal table of the nome $q$, Journal of the London Mathematical Society, vol. 5 (19.30) pp. 148-149. 